\title{
A low-cost repellent for malaria vectors in the Americas: results of two field trials in Guatemala and Peru Sarah J Moore ${ }^{1}$, Samuel T Darling*2, Moisés Sihuincha ${ }^{3}$, Norma Padilla ${ }^{4}$ and Gregor J Devine ${ }^{5}$
}

Address: ${ }^{1}$ Ifakara Health Research and Development Center, Ifakara, Tanzania, ${ }^{2}$ Puerta del Cielo Foundation, Canada/ Guatemala, ${ }^{3}$ Ministerio de Salud, Iquitos, Peru, ${ }^{4}$ Universidad del Valle, Guatemala City, Guatemala and ${ }^{5}$ Rothamsted Research, Harpenden, UK

Email: Sarah J Moore - smoore@ihrdc.or.tz; Samuel T Darling* - darlingi@telus.net; Moisés Sihuincha - Sihuincha@hotmail.com; Norma Padilla - npcz@cdc.gov; Gregor J Devine - greg.devine@bbsrc.ac.uk

* Corresponding author

Published: I August 2007

Malaria Journal 2007, 6:101 doi:10.1186/1475-2875-6-101
Received: 20 May 2007

Accepted: I August 2007

This article is available from: http://www.malariajournal.com/content/6/I/10I

(C) 2007 Moore et al; licensee BioMed Central Ltd.

This is an Open Access article distributed under the terms of the Creative Commons Attribution License (http://creativecommons.org/licenses/by/2.0), which permits unrestricted use, distribution, and reproduction in any medium, provided the original work is properly cited.

\begin{abstract}
Background: The cost of mosquito repellents in Latin America has discouraged their wider use among the poor. To address this problem, a low-cost repellent was developed that reduces the level of expensive repellent actives by combining them with inexpensive fixatives that appear to slow repellent evaporation. The chosen actives were a mixture of para-menthane-diol (PMD) and lemongrass oil (LG).

Methods: To test the efficacy of the repellent, field trials were staged in Guatemala and Peru. Repellent efficacy was determined by human-landing catches on volunteers who wore the experimental repellents, control, or $15 \%$ DEET. The studies were conducted using a balanced Latin Square design with volunteers, treatments, and locations rotated each night.

Results: In Guatemala, collections were performed for two hours, commencing three hours after repellent application. The repellent provided $>98 \%$ protection for five hours after application, with a biting pressure of $>100$ landings per person/hour. The 15\% DEET control provided lower protection at $92 \%(p<0.000 \mathrm{I})$. In Peru, collections were performed for four hours, commencing two hours after repellent application. The PMD/LG repellent provided $95 \%$ protection for six hours after application with a biting pressure of $>46$ landings per person/hour. The $20 \%$ DEET control provided significantly lower protection at $64 \%(p<0.000 \mathrm{I})$.

Conclusion: In both locations, the PMD/LG repellent provided excellent protection up to six hours after application against a wide range of disease vectors including Anopheles darlingi. The addition of fixatives to the repellent extended its longevity while enhancing efficacy and significantly reducing its cost to malaria-endemic communities.
\end{abstract}

\section{Background}

In 2005 , the WHO reported that $41 \%$ of malaria cases occur outside of Africa [1]. This marked a significant increase over their 2001 estimate of $13.6 \%$ [2] and reflects a growing awareness of the malaria problem beyond Africa. Greater recognition of this should encourage increased research on malaria vector control in other areas of the globe, an important consideration, as vectors in 
these regions generally exhibit behavior patterns that make them less susceptible to control measures shown to be effective in Africa, such as insecticide treated bednets (ITNs) and indoor residual spraying (IRS).

These behaviors include tendencies to [i] outdoor resting e.g. Anopheles darlingi [3] and Anopheles dirus [4]; ii) outdoor feeding e.g. Anopheles minimus [4], An. darlingi[3], and Anopheles sinensis [4]; and iii) significant feeding activity during early evening e.g. Anopheles albimanus [5], Anopheles nuneztovari [5], Anopheles farauti No.2 [6] and An. darlingi [7]. A study for implementing ITNs in four Latin America countries showed that $25 \%$ of An. albimanus in Nicaragua, $28 \%$ of Anopheles punctimacula in Ecuador, $57 \%$ of An. albimanus in Peru, and 30\% of $A n$. nuneztovari, also in Peru, fed before 9:00 pm, when people are still active and often still outdoors [8]. More recently, a case-control study in Colombia [9] showed that ITNs provided only $50 \%$ reduction in malaria. The authors attributed this to bites received when people were not sleeping under their nets. Even in areas where ITNs are considered to be highly effective malaria control tools, it appears that their introduction may have caused behavioral shifts among malaria vectors, with outdoor and early evening feeding becoming more frequent among them.

In such conditions, ITNs may be usefully supplemented by an effective insect repellent $[10,11]$. A recent household randomized trial in Pakistan [12] confirmed that the widespread provision of a repellent soap incorporating DEET and permethrin can reduce the risk of malaria by $>50 \%$. Moreover, an unpublished clinical trial in the Bolivian Amazon [13], with a 30\% para-menthane-diol (PMD) formulation, showed an $80 \%$ reduction in P. vivax in those using repellent and ITNs, compared to an ITN only group.

In countries burdened with malaria, nearly all families affected by the disease face severe economic pressure from loss of income and treatment costs. In fact, for the poorest families in Latin America these indirect costs may represent as much as $20 \%$ of annual household income $[14,15]$. In response to these conditions, a low-cost repellent lotion (LCR) was developed by S.T. Darling for distribution to malaria-endemic communities in the Americas.

This plant-based formulation (patent pending) incorporates two principal active ingredients: para-menthane-diol (PMD) and lemongrass oil (LG). PMD is derived from pine extractives or from lemon eucalyptus (Corymbia citriodora), a tree that is grown commercially in Latin America and China. PMD is a highly effective, broad-spectrum insect repellent [16-18] that has proven to lower malaria incidence where malaria transmission is maintained by An. darlingi [13]. Lemongrass oil, a distillate of Cymbopo- gon citratus or Cymbopogon flexuosus leaves, is traditionally used in many parts of the Americas to repel mosquitoes [19] and it is repellent to An. darlingi and other disease vectors (Moore et al., in preparation). To lower the cost of the repellent and maintain its efficacy, the PMD and LG actives were combined with some low-cost ingredients (fixatives) that extend the repellent effect by slowing the evaporation of volatile repellent actives [20]. The studies reported here were designed to measure whether such a repellent, containing lower amounts of actives than reported in previous studies, could prove its efficacy against malaria vectors and other mosquitoes at a muchreduced cost per application.

\section{Methods \\ Study Site A}

The field test was conducted near the Port of Champerico $\left(091^{\circ} 55^{\prime} \mathrm{W}, 14^{\circ} 18^{\prime} \mathrm{N}\right)$ on the Pacific coast of Guatemala, in June 2005. The estuaries and mangrove swamps that characterize the area are fed by rivers that descend from nearby volcanoes around Quezaltenango. The tests were conducted on a local finca - a farm where cattle are raised. The finca has a large permanent lagoon and swamp area that is a known An. albimanus breeding site. As An. albimanus feeds preferentially on cattle [21] it was ensured that livestock were enclosed 50 meters behind the volunteers, so that host-seeking mosquitoes emerging from the breeding site and attracted by the animal odours, would first encounter the collectors.

\section{Study site B}

The trial took place outside of Zungarococha, a small village near Iquitos, the capital of Loreto, Peru $\left(3.8^{\circ} \mathrm{S} 73.2^{\circ}\right.$ W). It was staged in late February 2006 in order to coincide with high An. darlingi populations, but while malaria transmission remained low. The region is lowland tropical forest with two tributaries of the Amazon River flowing through: the Nanay to the north, and the Itaya to the south. The volunteers collected mosquitoes in a sparsely wooded area that was situated between a group of houses and a lake, or cocha, fed by the river that is a permanent breeding site for An. darlingi.

\section{Test Repellents. Study A}

The following repellent formulations (\% by volume) were used: (1) C15 containing 15\% PMD (derived by acid modification of Corymbia citriodora; CAS: 42822-86-6; Chemian Technology Ltd), with LG (distilled from Cymbopogon citratus; CAS: 8007-02-1 The Essential Oil Company Ltd.), filler and fixative; (2) T15: 15\% PMD (derived by acid modification of Citronellal and recovery with aromatic hydrocarbons; CAS 42822-86-6; Takasago International Corporation), with LG, filler and fixative (patent pending); (3) T20: 20\% PMD (Takasago International Corporation), with LG, filler and fixative; (4) positive 
control: 15\% DEET ( $\mathrm{N}, \mathrm{N}$-diethyl-meta-toluamide, CAS 134-62-3; Sigma Aldridge) in ethanol; (5) negative control: filler mix.

\section{Test Repellents. Study B}

Slightly modified repellent formulations (\% by weight) were used: (1) PMD/LG containing 16\% PMD (Takasago International Corporation), with LG (Berje), filler and fixative (patent pending); (2) positive control: 20\% DEET (Sigma Aldridge) in ethanol (Sigma Aldridge); (3) negative control: 20\% mineral oil (ExxonMobil Corporation) in ethanol.

\section{Test Procedures. Study A and B}

Both studies were controlled, double-blinded, Latinsquare designs that utilized human-landing catches from treated or untreated volunteers in order to measure repellency. All solutions were placed in unmarked containers labelled by code. On any one night, human volunteers had both lower legs treated with either the PMD/LG candidate repellents or a positive or negative control at a rate of $0.002 \mathrm{ml} / \mathrm{cm}^{2}$ between the ankle and the knee. Volunteers' leg length and circumference were measured to calculate surface area, and the correct dose of treatment was measured using a micropipette. Repellent was then applied using a latex glove to minimize absorption of material by the hand of the volunteer. During the humanlanding catches, the volunteers wore shorts to the knee, work boots, and a loose bug jacket (ProBuy) to ensure that blood-seeking mosquitoes had access only to their lower legs. After midday, volunteers did not smoke, consume alcohol, or use soap when washing. This was intended to minimize variation in their headspace kairomones $[22,23]$.

The designated locations within the field sites were $10 \mathrm{~m}$ from each other and a minimum of $20 \mathrm{~m}$ from alternate sources of kairomones such as houses and livestock. As insect repellents act over a distance of less than a meter, and the maximum distance of host attraction of a single human to mosquitoes is $10 \mathrm{~m} \mathrm{[24]}$, this design minimizes the "relativity effect" wherein insects must choose between two hosts simultaneously. Mosquitoes were collected as soon as they landed on the exposed lower legs of the volunteers, but before probing of the skin commenced, using a mouth aspirator, flashlight, and collection vessel designed for this purpose. Collection vessels were changed each hour to provide hourly measures of repellence. Umbrellas were also provided to protect the volunteers from any rain showers that might wash away their repellent.

\section{Test Procedures. Study A}

The three repellents and two controls were applied to the five volunteers at $14.30 \mathrm{~h}$, and human-landing catches were performed at the field site for one hour before and one hour after sunset (1730-1930 h), when the evening mosquito biting is at its peak as shown by preliminary human-landing catches. The times chosen allowed an assessment of the protection afforded by the repellent over five hours while exposing volunteers to bites for only two hours. This helped minimize exposure and risk to the collectors. The study was a balanced $5 \times 5$ Latin-square design that required each volunteer to test each treatment five times over a period of 25 nights. Every evening, each individual was allocated one of five treatments, and sat in one of five allocated positions. Consequently, the volunteers changed position every five days.

\section{Test Procedures. Study B}

The repellent and two controls were applied at $16.00 \mathrm{~h}$, and man-landing collections were performed in the field, between 18.00 and $22.00 \mathrm{~h}$, as this is the time of peak $A n$. darlingi activity in the area. The times chosen allowed an assessment of the protection afforded by the repellent to be made for a period of six hours while exposing volunteers to bites for only four hours. This helped minimize exposure and risk to the collectors. The volunteers took a 15-minute break between 20.00 and $20.15 \mathrm{~h}$. The study was a $3 \times 3$ balanced Latin-square design that required each volunteer to test each treatment three times over a period of nine nights, with volunteers changing positions every third night. Due to unforeseen circumstances, one of the volunteers was replaced after four nights, and this was factored into the statistical analysis.

\section{Ethical Issues}

All volunteers were experienced at conducting man-landing catches. A form outlining procedure was given to the volunteers to ensure that they had full understanding of the potential risks of a study of this kind. In addition, each was given a chloroquine (Guatemala) or mefloquine (Peru) prophylaxis in accordance with WHO guidelines. Full ethical approval was obtained from Universidad del Valle, Guatemala (Study A); and from London School of Hygiene and Tropical Medicine Ethics Board and Instituto Nacional de Salud, Peru (Study B).

\section{Statistical Analysis}

Mosquitoes were maintained overnight and killed by cooling prior to identification the following morning. Data were normalized after transformation with natural $\log (\mathrm{x}+1)$, verified by Anderson-Darling Normality Test, and were analysed with General Linear Model (GLM) using SPSS 13 for Windows. The model measured the effect of position, individual, hour and treatment (as fixed factors) and day (as a random factor), on the transformed mosquito counts. Further post hoc testing of individual variables was performed using a Tukey's Honestly Significant Different test (Tukey's HSD). 


\section{Results Study A}

In 25 nights, 6,140 mosquitoes were captured comprising $55.6 \%$ Psorophora varipes (Coquillett) and 24.8\% Aedes ochlerotatus taeniorhynchus. The average number of mosquito landings on the negative control was 108 per person/hour and there was no significant difference in hourly numbers of landings in this treatment $(F=0.896$, d.f. $=1$, $p=0.345)$.

Each of the four repellents provided excellent protection from host-seeking mosquitoes, and the PMD/LG repellents provided $>97 \%$ protection up to five hours after application, with T15 and T20 providing 99\% protection. DEET (15\%) provided 92\% (Table 1). GLM analysis showed that there was a significant difference between the four repellents and the negative control, and between DEET and the three PMD based repellents; although there was no significant difference between the three PMD/LG repellents (Tukey's HSD, p < 0.0001) (Table 1). Sources of error in the experimental design were also investigated. There was no significant difference between the collection positions within the field site $(F=2.149$, d.f. $=4, p=$ $0.76)$, although individuals varied significantly in their mean attractiveness to mosquitoes/collection ability $(F=$ 6.73, d.f. $=4, p<0.0001)$.

Table I: Efficacy of 4 repellent formulations tested 4 and 5 hours after application during Study $A$ in Guatemala

\begin{tabular}{lccccc}
\hline & & \multicolumn{5}{c}{ Hours post application } \\
\hline Treatment & & 4 & 5 & Mean & $95 \%$ C.I. \\
\hline $\begin{array}{l}\text { Filler mix } \\
\text { control }\end{array}$ & AM & 115.52 & 100.48 & 108.00 & $55.13-96.39$ \\
& & & & & \\
& WM & 79.83 & 66.64 & $72.74^{\mathrm{a}}$ & \\
C15 & $\% \mathrm{P}$ & -- & -- & -- & \\
& AM & 1.28 & 5.04 & 3.16 & $0.46-01.38$ \\
& WM & 0.64 & 1.40 & $0.96^{\mathrm{b}}$ & \\
T15 & $\% \mathrm{P}$ & 98.89 & 94.98 & 97.07 & \\
& AM & 1.36 & 1.44 & 1.40 & $00.43-01.17$ \\
& WM & 0.79 & 0.72 & $0.76^{\mathrm{b}}$ & \\
T20 & $\% \mathrm{P}$ & 98.82 & 98.57 & 98.70 & \\
& AM & 0.84 & 1.84 & 1.34 & $0.28-00.97$ \\
& WM & 0.43 & 0.77 & $0.59 \mathrm{~b}$ & \\
I5 \% DEET & $\% \mathrm{P}$ & 99.27 & 98.17 & 98.76 & \\
& AM & 6.08 & 11.72 & 8.90 & $2.68-06.38$ \\
& WM & 3.63 & 4.87 & $4.21^{\mathrm{c}}$ & \\
& $\% \mathrm{P}$ & 94.74 & 88.34 & 91.76 & \\
\hline
\end{tabular}

$A M=$ arithmetic mean mosquito landings per person hour. WM = William's mean mosquito landings per person hour. Means followed by different letters are significantly different.

$\% \mathrm{P}=$ Percentage protection i.e. 100 - ((mosquito landings on treatment $\div$ mosquito landings on control $) \times 100$ ).

\section{Study B}

In nine nights, 2,358 mosquitoes were captured, of which $86 \%$ were An. darlingi. The average number of landings on the negative control was 46.28 per person/hour. There was no significant difference in the hourly number of mosquitoes captured from the control $(F=1.167$, d.f. $=3$, $p=0.326)$, or An. darlingi $(F=1.667$, d.f. $=3, p=0.179)$ which indicates that the repellents' efficacy did not significantly decline during the six hours of the test.

The PMD/LG repellent significantly outperformed DEET, providing an average of $95 \%$ protection six hours after application $(\mathrm{F}=128.8$, d.f. $=2, p<0.0001)$ (Table 2$)$. In contrast, $20 \%$ DEET provided an average of $64 \%$ protection over the duration of the trial. Sources of bias were investigated and there was no difference in the number of mosquitoes captured in the three positions within the field $(F=0.87$, d.f. $=2, p=0.422)$ or individual variation in attractiveness to mosquitoes/collection ability $(F=$ 1.492 , d.f. $=2, p=0.230$ ).

\section{Discussion and Conclusion}

In both field trials, the PMD/LG repellents with fixatives showed excellent efficacy against a broad range of mosquito species, with greater than expected longevity for a $15 \%$ PMD formulation. Also, in these trials the PMD/LG repellents showed greater efficacy than corresponding doses of DEET. This is an important point, as several other studies have shown PMD to have longevity similar to [17], or lower than [25], a corresponding dose of DEET. During a 2001 Bolivian field trial against An. darlingi where the biting pressure was 75 mosquito landings per person/ hour, a repellent containing $30 \%$ PMD in ethanol provided $97 \%$ protection, and 15\% DEET provided 85\% protection for four hours after application [17]. However, in the current Study A, the repellent containing half that concentration of PMD (T15) provided 99\% protection for five hours after application with a biting pressure of 108 mosquitoes per person/hour, compared to $92 \%$ for $15 \%$ DEET. In Study B, a repellent with 16\% PMD provided $95 \%$ protection for six hours after application, compared to $64 \%$ protection for $20 \%$ DEET.

It may be inferred from this that the addition of fixatives to the repellents tested in Guatemala and Peru slowed the release of repellent volatiles, thereby extending the repellents' duration and lowering its cost. Additionally, because PMD is the most costly ingredient in the repellent, the savings realized by a reduction in PMD content from $30 \%$ to $15 \%$ have reduced the cost of this disease prevention tool even more. There is much evidence that the indigenous poor are less likely to purchase ITNs and repellents, and more likely to rely upon cheaper, less effective methods of personal protection [26]. Therefore, mak- 
Table 2: Efficacy of 2 repellents tested 3 to 6 hours after application during study B in Peru.

\begin{tabular}{|c|c|c|c|c|c|c|c|c|c|c|c|c|c|}
\hline \multirow[b]{2}{*}{ Treatment } & & \multicolumn{6}{|c|}{ All mosquitoes Hours post application } & \multicolumn{6}{|c|}{ Anopheles darlingi Hours post application } \\
\hline & & 3 & 4 & 5 & 6 & Mean & 95 \% C.I. & 3 & 4 & 5 & 6 & Mean & $95 \%$ C.I. \\
\hline \multirow[t]{3}{*}{$20 \%$ oil control } & $\mathbf{A M}$ & 44.44 & 62.11 & 38.44 & 40.11 & 46.28 & $27.97-46.58$ & 31.11 & 54.78 & 34.11 & 35.44 & 38.86 & $20.05-37.64$ \\
\hline & WM & 34.52 & 53.05 & 31.46 & 29.27 & $36.13^{a}$ & & 22.10 & 44.60 & 23.05 & 24.79 & $27.52^{\mathrm{a}}$ & \\
\hline & $\% \mathbf{P}$ & - & - & - & - & - & & - & - & - & - & - & \\
\hline \multirow[t]{3}{*}{ PMD/LG Repellent } & AM & 1.33 & 1.78 & 1.67 & 4.78 & 2.39 & $0.73-2.10$ & I.II & 1.56 & 1.44 & 3.89 & 2.00 & $0.63-01.80$ \\
\hline & WM & 0.82 & 1.08 & 1.15 & 2.52 & $1.32^{\mathrm{b}}$ & & 0.66 & 0.93 & 1.05 & 2.17 & $1.14^{b}$ & \\
\hline & $\% \mathbf{P}$ & 97.01 & 97.13 & 95.66 & 88.08 & 94.84 & & 96.43 & 97.15 & 95.78 & 89.02 & 94.85 & \\
\hline \multirow[t]{3}{*}{$20 \%$ DEET } & $\mathbf{A M}$ & 15.22 & 19.78 & 13.89 & 18.44 & 16.83 & $10.17-17.25$ & 13.56 & 18.33 & 12.89 & 17.22 & 15.50 & $9.13-15.76$ \\
\hline & WM & 10.59 & 16.12 & 11.68 & 15.28 & $13.28 \mathrm{c}$ & & 9.59 & 14.96 & 10.59 & 13.59 & $12.02^{c}$ & \\
\hline & $\% \mathbf{P}$ & 65.75 & 68.15 & 63.86 & 54.03 & 63.63 & & 56.41 & 66.53 & 62.21 & 51.41 & 60.11 & \\
\hline
\end{tabular}

$A M=$ arithmetic mean mosquito landings per person hour.

WM = William's mean mosquito landings per person hour. Means followed by different letters are significantly different.

$\% \mathrm{P}=$ Percentage protection i.e. $100-(($ mosquito landings on treatment $\div$ mosquito landings on control $) \times 100)$.

ing the repellent available at the lowest cost possible could enhance user acceptance.

The potential benefit of this portable protection to antimalaria campaigns in the Americas can be seen in the economics of the 1998 malaria epidemic in Peru. It has been estimated that the total cost of treating malaria that year was $\$ 190$ per individual case [27]. That includes all associated state health expenses and the costs to the family for treatment, lost income, and death. Adjusted for inflation, that is approximately $\$ 250$ in 2007 . However, the estimated annual cost per person for the repellent intervention (coverage during the 7 -month transmission season at $\$ 0.024 /$ day) is $\$ 5.00$. That is $2 \%$ of the estimated total cost of treatment today. Consequently, the PMD/LG repellent may be an excellent candidate for incorporation into existing vector control strategies.

If such economies could be achieved in Peru, while reducing the disease burden from malaria, this model might be applicable to other regions where early evening biting is problematic. Essentially, the model for "repelling" malaria proposes the following: where the crepuscular feeding behavior of the most significant malaria vectors is already established, or where it may be shifting to early evening biting or outdoor resting [28] (from selection due to IRS and/or ITN use), it may be possible to achieve a reduction of $60 \%$ in malaria cases [13]. This could be achieved by saturation of at-risk communities (to avoid diversion of malaria vectors to non-users) with high-efficacy LCRs that are affordable and aromatically attractive to the indigenous poor. Combined with ITNs, the reduction could be substantially greater.

A five-month Phase 3 study, currently under way in the Peruvian Amazon, is providing an opportunity to measure the parameters of this model on 16 population clus- ters. With 3,300 subjects divided into three cohorts (Repellent Only, Repellent + ITNs, and No Intervention), this community-wide study marks the first time that the effect of a Repellent Only intervention on malaria rates has been measured in a discrete population group in the tropics. If the LCR's demonstrated capacity to repel malaria vectors leads to a measurably significant reduction in the infection rate, the Puerta del Cielo Foundation will begin distributing repellents and treated bednets at cost to poor malaria-endemic communities throughout the Peruvian Amazon.

\section{Competing interests}

The research that appears in this manuscript was funded and directed by S.T. Darling with organizational support from Puerta del Cielo, a Guatemalan non-profit foundation. A patent in Mr. Darling's name is pending on one of the repellent formulations tested in these trials. When approved, this patent will be conveyed to an NGO that will distribute the repellent at cost (not-for-profit) to malaria-endemic communities in the Americas and elsewhere. Mr. Darling will not profit from its development or sale. Under Mr. Darling's direction, Sarah Moore was paid to carry out specific tasks related to his repellent research. The remaining co-authors gave their services freely to this effort and, therefore, retained their independence from the funder.

\section{Authors' contributions}

SJM designed the study protocols, supervised the repellent trials, collected and analysed the data, and co-authored the manuscript. STD directed the repellent research, designed the repellent, co-authored the manuscript, and was responsible for editing. MS: provided translation and logistical support in Iquitos, Peru. NP provided translation and organizational help through CDC/MERTU and Universidad del Valle in Guatemala. GJD implemented 
the study protocols in Peru, guided them through ethics committees there, and contributed to editing the manuscript.

\section{Acknowledgements}

We are grateful to the Puerta del Cielo Foundation for the organizational support they provided. We are also grateful for the enthusiasm and expertise of the collectors in Guatemala and Peru who made this study possible. Guatemala: Juan Garcia, Edwin Escobar, Emilse Barillasis, Cristian Garcia. We would like to thank Sra. Garcia for her hospitality to SJM and the collection team, Don Mateo for use of the field site, and CDC/MERTU in Guatemala for their logistical support. Peru: Andreas Rojas, Carlos Estrella, Wagner Orellana, Carlos Valderama, Carlos Rojas. We thank the DISA Loreto Staff, also Carlos Alvarez and Elvira Zamora of the Laboratorio de Salud Publica, Iquitos, and the residents of Zungaracocha. Special thanks are extended to Nigel Hill (LSTMH) for his assistance in applying for ethical clearance and guidance on study design, and to Patricia Aiyenuro for providing mosquitoes for use in preliminary laboratory studies. Ingredients were generously provided by Berje, Chemian Technologies Ltd, ExxonMobil Corporation, Takasago International Corporation, and The Essential Oil Co Ltd.

\section{References}

I. Hay SI, Guerra CA, Tatem AJ, Noor AM, Snow RW: The global distribution and population at risk of malaria: past, present, and future. Lancet Infect Dis 2004, 4:327-336.

2. WHO: World malaria report 2005. (WHO/HTM/MAL/ 2005.I / 02) [http://www.rbm.who.int/wmr2005/html/l-2.htm] Geneva: World Health Organization and UNICEF

3. Tadei WP, Dutary Thatcher B: Malaria vectors in the Brazilian Amazon: Anopheles of the subgenus Nyssorhynchus. Rev Inst Med Trop Sao Paulo 2000, 42:87-94.

4. Trung HD, Bortel WV, Sochantha T, Keokenchanh K, Briet OJ, Coosemans M: Behavioural heterogeneity of Anopheles species in ecologically different localities in Southeast Asia: a challenge for vector control. Trop Med Int Health 2005, 10:25I-262.

5. Rubio-Palis $\mathrm{Y}$, Curtis CF: Biting and resting behaviour of anophelines in western Venezuela and implications for control of malaria transmission. Med Vet Entomol 1992, 6:325-334.

6. Benet A, Mai A, Bockarie F, Lagog M, Zimmerman P, Alpers MP, Reeder JC, Bockarie MJ: Polymerase chain reaction diagnosis and the changing pattern of vector ecology and malaria transmission dynamics in Papua New Guinea. Am J Trop Med Hyg 2004, $71: 277-284$.

7. Harris AF, Matias-Arnez A, Hill N: Biting time of Anopheles darlingi in the Bolivian Amazon and implications for control of malaria. Trans R Soc Trop Med Hyg 2006, 100:45-47.

8. Kroeger A, Meyer R, Mancheno M, Gonzalez M, Pesse K: Operational aspects of bednet impregnation for commuity-based malaria control in Nicaragua, Ecuador, Peru and Colombia. Trop Med Int Health 1997, 2:589-602.

9. Alexander N, Rodriguez M, Perez L, Caicedo JC, Cruz J, Prieto G, Arroyo JA, Cotacio MC, Suarez M, F DLH, Hall AJ: Case-control study of mosquito nets against malaria in the Amazon region of Colombia. Am J Trop Med Hyg 2005, 73: I40-I 48.

10. Costantini C, Badolo A, llboudo-Sanogo E: Field evaluation of the efficacy and persistence of insect repellents DEET, IR 3535 and KBR 3023 against Anopheles gambiae complex and other Afrotropical vector mosquitoes. Trans R Soc Trop Med Hyg 3535, 98:644-652.

II. Hii JLK, Kanai L, Foligela A, Kan KP, Burkot TR, Wirtz RA: Impact of permethrin-impregnated bednets compared with DDT house-spraying against malaria transmission by Anopheles farauti and An. punctulatus in the Solomon Islands. Med Vet Entomol 1993, 7:333-338.

12. Rowland M, Downey G, Rab A, Freeman T, Mohammad N, Rehman H, Durrani N, Reyburn H, Curtis C, Lines J, Fayaz M: DEET mosquito repellent provides personal protection against malaria: a household randomized trial in an Afghan refugee camp in Pakistan. Trop Med Int Health 2004, 9:335-342.

13. Hill N, Lenglet A, Matias-Arnez A, Carneiro I: Clinical evaluation of combined use of ITNs and plant-based insect repellents to control malaria in Bolivia. Malaria Centre Report 2005:50-51 [http://www.lshtm.ac.uk/malaria/annualreport/report20042005.pdf]. London School of Hygiene and Tropical Medicine

14. Vosti S: Malaria among gold miners in Southern Para, Brazil: estimates of determinants and individual costs. Soc Sci Med 1990, 30:1097-II05.

15. Pang LW, Piovesan-Alves F: Economic advantage of a community-based malaria management programme in the Brazilian Amazon. Am J Trop Med Hyg 200I, 65:883-886.

16. Trigg JK: Evaluation of a eucalyptus-based repellent against Anopheles spp. in Tanzania. J Am Mosq Control Assoc 1996, I 2:243-246

17. Moore SJ, Lenglet A, Hill N: Field evaluation of three plantbased insect repellents against malaria vectors in Vaca Diez Province, the Bolivian Amazon. J Am Mosq Control Assoc 2002, 18:107-110.

18. Schreck CE, Leonhardt BA: Efficacy assessment of Quwenling, a mosquito repellent from China. J Am Mosq Control Assoc 1991, 7:433-436.

19. Sears R: An ethnobotanical survey of insect repellents in Brazil. TRI News 1996, I 5:8-10.

20. Rutledge LC, Gupta RK, Mehr ZA, Buescher MD, Reifenrath WG: Evaluation of controlled-release mosquito repellent formulations. J Am Mosq Control Assoc 1996, 1 2:39-44.

21. Grieco JP, Achee NL, Andre RG, Roberts DR: Host feeding preferences of Anopheles species collected by manual aspiration, mechanical aspiration, and from a vehicle-mounted trap in the Toledo District, Belize, Central America. J Am Mosq Control Assoc 2002, 18:307-315.

22. de Jong R, Knols BG]: Selection of biting sites on man by two malaria mosquito species. Experientia 1995, 51:80-84.

23. Shirai OT, Tsuda T, Kitagawa S, Naitoh K, Seki K, Kamimura K, Morohashi M: Alcohol ingestion stimulates mosquito attraction. J Am Mosq Control Assoc 2002, 18:91-96.

24. Gillies MT, Wilkes TJ: Range of attraction of single baits for some West-African mosquitoes. Bulletin of Entomological Research 1970, 60:225-235.

25. Barnard DR, Bernier UR, Posey KH, Xue RD: Repellency of IR 3535 KBR3023, para-menthane-3,8-diol, and DEET to black salt marsh mosquitoes (Diptera: Culicidae) in the Everglades National Park. J Med Entomol 3535, 39:895-899.

26. Worrall $E$, Basu $S$, Hanson $\mathrm{K}$ : Is malaria a disease of poverty? A review of the literature. Tropical Medicine and International Health 2005, 10:1047-1059.

27. MINSA/USAID: Impacto económico de la Malaria en el Perú. 2000:122 [http://www.minsa.gob.pe/pvigia/inst.apoyoMalaria\%5CpublicacionESP.pdf]. Lima, Perú: Ministerio de Salud

28. Charlwood JD: Biological variation in Anopheles darlingi (Root). Memorias de Instituti Oswaldo Cruz 1996, 91 1:391-398.

Publish with Biomed Central and every scientist can read your work free of charge

"BioMed Central will be the most significant development for disseminating the results of biomedical research in our lifetime. "

Sir Paul Nurse, Cancer Research UK

Your research papers will be:

- available free of charge to the entire biomedical community

- peer reviewed and published immediately upon acceptance

- cited in PubMed and archived on PubMed Central

- yours - you keep the copyright 